\section{Safety first - sedate and shock}

To the editor: Mitchell and Lazarenko recently authored a diagnostic challenge in the November 2008 issue of CJEM. ${ }^{1}$ We thank the authors for providing this worthy topic for discussion and congratulate them on their publication. However, with due respect, we take considerable issue with several of the conclusions reached in this article and ultimately with the answer to the diagnostic challenge. Although it is alluded to as a possible treatment twice in the discussion, we assert that the safest approach for the emergency physician is to treat all wide complex tachycardia as ventricular tachycardia (VT).

As with many situations in emergency medicine, when a patient presents with an unstable wide complex dysrhythmia, we are forced to make decisions without the benefit of much of the information referenced within the article. The assessment for the presence of cannon atrial waves or variability of the first heart sound requires clinical acumen well beyond even the expert emergency physician. In fact, the additional criterion of variability in beat-tobeat systolic blood pressure requires the placement of an arterial catheter, which is impractical and unnecessary in many instances.

Electrolytes are rarely available and toxicology screens are rarely helpful. The authors state that the toxicology screen was negative, which we believe to be relatively unhelpful in this setting (and in many others), as this test is insufficiently sensitive or specific for the presence of tricyclic antidepressants or any other sodium channel blockers that may be important toxicological causes of wide complex dysrhythmia.

In a 45-year-old patient, the authors acknowledge that even if based solely on the criterion of age, one ought to assume this is VT. Although not polymorphic, the addition of sotalol would increase the pretest likelihood of VT as well.

If indeed the authors' logic is correct and the remaining differential includes supraventricular tachycardia (SVT) with aberrancy by way of an accessory pathway, one still ought to avoid adenosine in these instances. There are several reports of ventricular fibrillation associated with adenosine therapy, in both Wolff-Parkinson-White syndrome $^{2-4}$ and in the treatment of VT. ${ }^{5}$ The safest approach in this setting would be electrical cardioversion or, in a stable patient, procainamide. ${ }^{6}$

We thank the authors for their comments regarding the application of the Brugada criteria. The originally reported sensitivity and specificity were $95.7 \%$ and $96.5 \%{ }^{7}$ which have never been externally reproduced. Two reports have failed to replicate these numbers in the hands of cardiologists or emergency physicians, suggesting even less broad applicability in the emergency setting. ${ }^{8,9}$ These numbers are not sufficient to exclude VT, particularly when considering verapamil or diltiazem as a potential therapy.

Even if these numbers were acceptable to some practitioners (which is where our concerns lie), life-threatening consequences of misdiagnosing VT as SVT with aberrancy are unacceptable when the therapy of choice for VT works equally well for SVT with aberrancy with very little added potential for harm. The authors correctly suggest that when the diagnosis is uncertain, one should treat the dysrhythmia as VT. Arguably, based on the information provided by these 2 papers, ${ }^{89}$ one can rarely be certain of the diagnosis and should routinely proceed with the treatment of wide complex tachycardia as VT.

The lasting impression of the article is one of using a medical treatment that has grave risks associated with its use. We suggest that when an article is published one should concern themselves with the message that is delivered to the typical emergency physician, and therefore should project a message that is safe and effective.

In this case, no one should close the journal thinking that through cannon atrial waves associated with beat-tobeat systolic variability and Brugada criteria that they can differentiate these 2 entities in the chaos of the emergency department. Let us put the safety of our patients first and the vanity of superior academic skills second.

Sedate and shock this rhythm - be safe.

\section{Andrew Healey, MD}

PGY5, Emergency Medicine/Critical Care Resident, McMaster University, Hamilton, Ont.

Mark Mensour, MD, FCFP

Assistant Professor, Emergency

Medicine, Northern Ontario School

of Medicine, Huntsville, Ont.

Thomas Marshall, MD

Staff Emergency Physician, St. Joseph's Healthcare, Hamilton, Ont.

\section{References}

1. Mitchell J, Lazarenko G. Wide QRS complex tachycardia. CJEM 2008;6: 572-3.

2. Gupta AK, Shah CP, Maheshwari A, et al. Adenosine induced ventricular fibrillation in Wolff-Parkinson-White syndrome. Pacing Clin Electrophysiol 2002;25:477-80.

3. Gallagher JJ, Sealy WC, Kasell J, et al. Multiple accessory pathways in patients with the pre-excitation syndrome. Circulation 1976;54:571-91.

4. Exner DV, Muzyka T, Gillis AM. Proarrhythmia in patients with the Wolff-Parkinson-White syndrome after standard doses of intravenous adenosine. Ann Intern Med 1995;122:351-2.

5. Parham WA, Mehdirad AA, Biermann $\mathrm{KM}$, et al. Case report: adenosine induced ventricular fibrillation in a patient with stable ventricular tachycardia. J Interv Card Electrophysiol 2001;5:71-4.

6. Tijunelis MA, Herbert ME. Myth: 
intravenous amiodarone is safe in patients with atrial fibrillation and WolffParkinson-White syndrome in the emergency department. CJEM 2005;7: 262-5.

7. Brugada P, Brugada J, Mont L, et al. A new approach to the differential diagnosis of a regular tachycardia with a wide QRS complex. Circulation 1991; 83:1649-59.

8. Isenhour JL, Craig S, Gibbs M, et al. Wide-complex tachycardia: continued evaluation of diagnostic criteria. Acad Emerg Med 2000;7:769-73.

9. Herbert ME, Votey SR, Morgan MT, et al. Failure to agree on the electrocardiographic diagnosis of ventricular tachycardia. Ann Emerg Med 1996;27:35-8.

\section{[The authors respond]}

We thank Drs. Healey, Mensour and Marshall for their interest in our diagnostic challenge published in the November 2008 issue of CJEM. ${ }^{1}$ They summarize their comment on our article by stating, "The safest approach for the emergency physician is to treat all wide complex tachycardias as ventricular tachycardia (VT)." As we indicated, ${ }^{1}$ we essentially agree with this point of view, with one notable exception. We welcome the opportunity to further discuss this distinction.

The correspondents posit that "one can rarely be certain of the diagnosis and should routinely proceed with the treatment of wide complex tachycardia as VT" by sedating and shocking the patient. We contend that, in treating a stable patient, supraventricular tachycardia (SVT) with aberrant conduction can be distinguished from VT with sufficient frequency to warrant the effort. Although the default position in uncertain cases is to treat as VT, when the rhythm is SVT with aberrant conduction, the American Heart Association guidelines for emergency care ${ }^{2}$ recommend adenosine as first-line therapy.

Our diagnostic challenge included an electrocardiogram (ECG) showing a regular, wide QRS tachycardia with typical left bundle branch block (LBBB) morphology. The differential diagnosis in this case includes VT and SVT with LBBB. The latter includes sinus tachycardia, true atrial tachycardia, atrioventricular (AV) nodal re-entry and orthodromic reciprocating tachycardia in a patient with Wolff-Parkinson-White (WPW) syndrome (down the normal conduction pathway and up the accessory AV connection); each with LBBB. This tachycardia has less than a $10 \%$ chance of being VT, ${ }^{3}$ and when it is VT, it is often adenosine-sensitive right ventricular outflow tract VT. ${ }^{4}$

Dr. Healey and colleagues suggest that "one still ought to avoid adenosine in these instances." As quoted by the correspondents, adenosine may be dangerous in WPW syndrome patients with pre-excited atrial fibrillation by increasing the frequency of accessory connection conduction of atrial fibrillation, thereby predisposing the patient to the development of ventricular fibrillation (VF). In the cases cited by Dr. Healey and coworkers that converted to VF after receiving adenosine,, 5 all initially presented with preexcited atrial fibrillation. By definition, such rhythms are grossly irregular with atypical aberrancy that should not be mistaken for a regular SVT. This is not meant to imply that adenosine for regular SVT is without risk. One rarely reported risk is conversion of a regular SVT to atrial fibrillation which, in the presence of manifest WPW syndrome, could be conducted at a rapid rate. ${ }^{6}$ Our patient did not have manifest WPW syndrome, as confirmed by history and by previous and follow-up ECGs. We could find only 1 report of VT degenerating into VF after adenosine was given, ${ }^{7}$ and reports of VT degenerating into VF in the absence of adenosine are numerous. ${ }^{8}$ Indeed, adenosine has been recommended as a safe diagnostic aid that can be used to distinguish regular SVT with aberrant conduction from VT. ${ }^{9-11}$ Thus the probability of a serious complication from adenosine in the setting of a regular wide QRS tachycardia is remote ${ }^{12}$ and does not support the correspondents' statement that adenosine in this setting "has grave risks associated with its use." Furthermore, alternative treatments, such as intravenous procainamide and direct current cardioversion, have complications of their own, including ventricular proarrhythmia. Finally, in our patient, treatment with procainamide was not advised as the patient was already on sotalol. Combining 2 agents known to prolong the QT interval might elicit torsades de pointes.

The correspondents conclude by stating, "Let us put the safety of our patients first and the vanity of superior academic skills second." We agree with the first statement and consider the second to be an unfortunate choice of words. Rather than a routine "sedate and shock" approach, we offer an alternative: "think and link" the treatment of a tachydysrhythmia to its evident pathophysiology. Nevertheless, what is ultimately done will depend on local standards of care and the confidence of the clinician in decision-making in the emergency department.

\section{Jason Mitchell, BSc}

Medical Student, University of Calgary Health Region, Calgary, Alta.

\section{Gerald Lazarenko, MD}

Clinical Assistant Professor, University of Calgary, Calgary, Alta.

\section{References}

1. Mitchell J, Lazarenko G. Wide QRS complex tachycardia. CJEM 2008;6: 572-3.

2. ECC Committee; Subcommittees and Task Forces of the American Heart Association. 2005 American Heart Association Guidelines for Cardiopulmonary Resuscitation and Emergency Cardiovascular Care. Circulation 2005;112: IV1-203.

3. Griffith MJ, Garratt CJ, Mounsey P, et al. Ventricular tachycardia as default diagnosis in broad complex tachycardia. 